\title{
Philosophiques
}

\section{La pensée philosophique médiévale. Contribution canadienne} (1960-1973)

\section{Albert-M. Landry}

Volume 1, numéro 2, octobre 1974

URI : https://id.erudit.org/iderudit/203017ar

DOI : https://doi.org/10.7202/203017ar

Aller au sommaire du numéro

Éditeur(s)

Société de philosophie du Québec

ISSN

0316-2923 (imprimé)

1492-1391 (numérique)

Découvrir la revue

Citer cet article

Landry, A.-M. (1974). La pensée philosophique médiévale. Contribution canadienne (1960-1973). Philosophiques, 1(2), 111-139.

https://doi.org/10.7202/203017ar d'utilisation que vous pouvez consulter en ligne.

https://apropos.erudit.org/fr/usagers/politique-dutilisation/ 


\title{
BULLETIN
}

\section{LA PENSÉE PHILOSOPHIQUe MÉDIÉVALE CONTRIBUTION CANADIENNE 1960-1973}

\author{
par Albert-M. LANDRY, O.P.
}

Un relevé complet des recherches et des travaux consacrés par les chercheurs et les Centres ou Instituts d'études médiévales canadiens au vaste champ d'enquête que constitue la période médiévale, révélerait sans doute que leurs contributions n'ont cessé d'augmenter en quantité, en qualité et en variété depuis les années où les études médiévales ont connu leur premier grand essor. Dans les quelques pages qui suivent, on voudrait présenter en une modeste vue d'ensemble - qui ne peut d'ailleurs se présenter comme exhaustive - ce que fut, dans le seul domaine de la pensée philosophique médiévale, la contribution canadienne en travaux et recherches au cours des années 1960-1973 ${ }^{1}$.

1. Sous le mot «canadien» sont inclus dans le présent BULLETIN les ouvrages et travaux aussi bien des chercheurs et auteurs canadiens oeuvțant ou au Canada ou à l'étranger que des professeurs et chercheurs d'autres pays invités à travailler au Canada temporairement ou en permanence et ayant publié dans des revues ou des collections canadiennes. - L'expression «pensée philosophique médiévale» indique bien les cadres à l'intérieur desquels on a dû se tenir. Ont donc été passés sous silence de nombreux travaux ayant une portée doctrinale mais qui par leur objet faisaient partie des disciplines théologiques. - Pour des renseignements sur les divers Centres et Instituts d'études médiévales au Canada voir Albert-M. LANDRY, O.P., Chroniques nationales, IV, 1 - Canada, dans BSIEPM, 13 (1971), pp. 166-182; Joseph ROLLAND, La section canadienne de la Commission léonine, dans $R U O, 30,1(1960)$, pp. 57*-58*. - Voici les sigles utilisés dans le présent BULLETIN : BSIEPM $=$ Bulletin de la société internationale pour l'étude de la pbilosopbie médiévale (Louvain) ;CAG $=$ 
INSTRUMENTATION. - Mentionnons d'abord brièvement les travaux qui, à un titre ou à un autre, entrent dans la catégorie des ouvrages constituant les instruments de travail indispensables au chercheur. Bibliothèques. - Le Pontifical Institute of Mediaeval Studies de Toronto vient de constituer un «Dictionary Catalogue» de sa bibliothèque spécialisée dans lequel sont décrits quarante mille monographies, quarante mille micro-photogrammes et le contenu de cent vingt-neuf périodiques. Les quelque 80,000 fiches de ce catalogue portant sur l'histoire de l'art, 'les études byzantines, les droits civil et canonique, l'histoire de l'Église, la patristique, la philosophie, la théologie et les langues vernaculaires, sont reproduites en cinq volumes reliés, à raison de 21 fiches par page de $10 \times 14$ sur papier spécialement choisi pour ses qualités de permanence et de durabilité. Est-il besoin d'insister sur l'utilité d'un tel instrument pour ceux qui travaillent sur l'une ou l'autre des disciplines médiévales ${ }^{2}$. C'est aussi au chercheur sur place, mais tout autant à celui qui de loin voudrait avoir une idée de ce que sont les Archives du Vatican que L.E. Boyle pensait en publiant $A$ Survey of the $V$ atican Archives and of Its Mediaeval Holdings ${ }^{3}$. Une biblographie choisie et un Index général du texte et de la bibilographie tlle-même complètent cette introduction aux archives vaticanes dont on n'a pas à faire ressortir la richesse pour l'étude de l'histoire. Répertoires de manuscrits. - En collaboration avec $\mathrm{H}$. F. Dondaine et d'autres membres de la Commission léonine pour l'édition des oeuvres de saint Thomas, H. V. Shooner a publié les deux premiers tomes du catalogue descriptif et critique des

Conférences Albert-le-Grand (Montréal) ; LTP = Laval théologique et philosophique (Québec); $M S t=$ Mediaeval Studies (Toronto); PIEM $=$ Publications de l'Institut d'études médiévales (Montréal); RUO = Revue de l'Université d'Ottawa (Ottawa); $\mathrm{ScE}=$ Sciences ecclésiastiques devenues en 1968 Science et Esprit (Montréal) ; $S M R=$ Studia Montis Regii (Montréal); $S t T=$ Studies and Texts (Toronto).

2. Pontifical Institute of Mediaeval Studies, Toronto, Canada. Dictionary Catalogue of the Library, 5 vol., Boston, 1972. Pour plus de détails, voir BSIEPM, 14 (1972), p. 129.

3. Leonard E. BOYLE, O.P., A Survey of the Vatican Archives and of lts Mediaeval Holdings (Subsidia mediaevalia, I), Toronto, 1972, $250 \mathrm{pp}$. 
manuscrits des oeuvres de saint Thomas ${ }^{4}$. Le catalogue comprendra cinq tomes dans lesquels les manuscrits doivent être décrits selon leur appartenance aux diverses bibliothèques, ellesmêmes classifiées selon l'ordre alphabétique des villes où elles se trouvent. La critique a accueilli le premier tome avec grands éloges et souhaite vivement voir l'oeuvre se poursuivre et se terminer dans un délai aussi bref que possible. M. Shooner avait déjà, en collaboration avec Th. Kappeli, pourvu à la publication du catalogue des manuscrits médiévaux se trouvant à Saint-Dominique de Dubvronik ${ }^{5}$. De son côté, J. A. Weisheipl a publié une liste préliminaire de manuscrits contenant des écrits de dix-neuf maîtres de Merton College de la première moitié du XIVe siècle ${ }^{6}$, tandis que N. M. Haring a fait l'inventaire des manuscrits où se trouvent des commentaires du credo pseudo-athanasien, sa liste provisoire comprenant vingt-trois commentaires anonymes et ceux de seize auteurs connus des XIe-XVe siècles ${ }^{7}$. B. Allard, enfin, qui prépare l'édition critique et l'étude philosophique du $D e$ anima de Guillaume d'Auvergne, a pu corriger et augmenter les renseignements fournis sur cet auteur par P. Glorieux dans son Répertoire des maîtres du XIIIe siècle ${ }^{8}$. Bibliograpbies. - Le Center for Mediaeval Studies de l'Université de Toronto a pris l'initiative de faire paraitre une collection qui portera le titre de Toronto Mediaeval Bibliograpbies et qui sera consacrée aux di-

4. Codices manuscripti operum Thomae de Aquino recensuerunt H. F. DONDAINE et $\mathrm{H}$. V. SHOONER cooperantibus sociis Commissionis Leoninae (Editores operum sancti Tbomas de Aquino, 2). T. I : Autograpba et Bibliotbecae A-F, Rome, 1967, XVI-384 pp., 12 pl.; T. II. Bibliotbecae G-M, Rome, 1973, XIII-435 pp., Suppl. 1*-8* pp.

5. Les manuscrits médiévaux de Saint-Dominique de Dubvronik. Catalogue sommaire compilé par Th. KAEPPELI et $\mathrm{H}$. V. SHOONER (Institutum bistoricum FF. Praedicatorum, XVII), Rome, 1965, 146 pp.

6. James A. WEISHEIPL, Repertorium Mertonense, dans MSt, (1969), pp. $174-224$

- Nicholas M. HARING, Commentaries on the Pseudo-Athanasian Creed, dans MSt, XXXIV (1972), pp. 208-252.

8. Baudoin ALLARD, Additions au Répertoire des Maitres en tbéologie de Paris au XIIle siècle. A propos de Guillaume d'Auvergne, dans BSIEPM, 5 (1963), pp. 147-148; Idem Nouvelles additions et corrections au "Répertoire» de Glorieux. A propos de Guillaume d'Auvergne, ibidem, 10-12 (1968-1970), pp. 212-224.

9. James J. MURPHY, Medieval Rbetoric. A Select Bibliograpby (Toronto Medieval Bibliographies, 3), Toronto, 1971, XVIII-102 pp. 
verses disciplines médiévales. Ayant un intérêt philosophique dans cette collection sont celle de J.J. Murphy sur la rhétorique médiévale ${ }^{9}$ et celle de J.E. Murdoch sur les sciences exactes et la philosophie de la nature au moyen âge (en préparation). Sans se présenter comme une bibliographie proprement dite, l'Introduction à l'bistoire de la littérature théologique de la scolastique naissante de A. M. Landgraf, qui vient de paraître en édition française complètement refondue et mise à jour, est une mine de renseignements bibliographiques sur les maitres, les oeuvres et les diverses écoles du XIIe et du début du XIIIe siècle ${ }^{\mathbf{1 0}}$. Comme bibliographie consacrée à un seul auteur, on en relève une provisoire, celle de A. D. Menut, consacrée à Nicole Oresme ${ }^{11}$. Latin médiéval. - Il convient de signaler, encore dans ce secteur consacré à l'instrumentation, l'utile article de A. Pattin sur le vocabulaire philosophique médiéval où l'auteur conclut que, grâce à la formation de termes nouveaux pour tenter d'exprimer adéquatement certains concepts, le latin médiéval est devenu une langue technique de la pensée abstraite ${ }^{12}$. Editions de textes et traductions. - L'effort, dans ce secteur, a été remarquable et on saura gré aux éditeurs de textes d'avoir déployé leur activité et leur patience à publier en éditions modernes des oeuvres dont l'existence au seul état manuscrit retardait la connaissance et l'utilisation. La plupart de ces éditions, dont quelques-unes se présentent comme des éditions critiques, sont accompagnées d'introductions, de notes ou d'études plus ou moins fouillées. On ne peut guère en fournir ici plus qu'une simple énumération où l'on suivra l'ordre chronologique: de N. M. Hàring, l'édition d'extraits de quatre commentaires sur le De consolatione philosophiae contenus dans le manuscrit Heiligenkrenz 130 (XIIe siècle) et

10. Artur Michael LANDGRAF, Introduction à l'bistoire de la littérature théologique de la scolastique naissante, Edition française par les soins de Albert-M. LANDRY, O.P., traduction de l'allemand par Louis-B. GEIGER, O.P. (PIEM, XXII), Montréal-Paris, 1973, 212 pp.

11. Albert-D. MENUT, $A$ Provisional Bibliograpby of Oresme's Writings, dans MSt, XXVIII (1966), pp. 279-299; idem A Provisional Bibliography of Oresme's Writing. A Supplementary Note, ibid., XXXI (1969), pp. 346-347.

12. A. PATTIN, Notes sur le vocabulaire philosopbique médiéval, dans RUO, 33,4 (1963) , pp. $193^{*}-213^{*}$. 
qui pourraient être, le premier de Jean Scot Erigène, le deuxième, un texte du groupe Anonymus Erfurtensis, le troisième, la rélaction d'une glose de Remi d'Auxerre et le quatrième, un texte remontant à Guillaume d'Auxerre ${ }^{13}$; - de V. Brown, l'édition du Liber de natura deorum d'un auteur anonyme du XIIe siè$\mathrm{cle}^{14}$; - de R. D. Crouse, l'édition critique du texte complet du De neocosmo d'Honorius d'Autun ${ }^{15} ;-$ de B. Stock, l'édition d'un court texte anonyme de cosmologie influencé par Hugues de Saint-Victor ${ }^{16}$; - de N. M. Haring, un ensemble de textes de Gilbert de Poitiers et de membres de son école, soit le commentaire sur le credo pseudo-athanasien de Gilbert d'après le seul manuscrit connu Klosterneuberg, Stiftsbibliothek, ms $815^{17}$, le De discretione animae, spiritus et mentis dont Haring attribue la paternité à Gilbert et non plus à Achard de Saint-Victor ${ }^{18}$, cinq textes de commentaires de Gilbert sur les Opuscula sacra de Boèce avec, en appendice, le texte même de ces opuscules utilisé par Gilbert ${ }^{19}$, les lettres de Pierre de Vienne et de Hugues de Honau adressées à Hugues Ethérien pour obtenir des éclaircissements sur les concepts de nature et de personne ${ }^{20}$, un Liber de ignorantia de Hugues de Honau ${ }^{21}$, une compilation de douze textes patristiques d'après le manuscrit Paris. Arsenal 1117 B

13. Nicholas M. HARING, Four Commentaries on the De Consolatione Philosopbiae in MS Heiligenkreuz 130, dans MSt, XXXI (1969), pp. 287-316.

14. Virgina BROWN, An Edition of an Anonymous Twelfth-Century Liber de natura deorum, dans MSt, XXXIV (1972), pp. 1-70.

15. HONORIUS D'AUTUN, De Neocosmo. A Critical Edition of the Text with Introduction and Notes, by Robert Darwin CROUSE, Cambridge (Mass.), 1970, VIII-252 pp.

16. Brian STOCK, Hugh of St. Victor, Bernard Silvester and MS Trinity College, Cambridge, 0. 7. 7, dans MSt, XXXIV (1972), pp. 152-173.

17. Nicholas M. HARING, A Commentary on the Pseudo-Atbanasian Greed by Gilbert of Poitiers, dans MSt, XXVII (1965), pp. 23-53.

18. IDEM, Gilbert of Poitiers, Autbors of the De discretione animae, spiritus et mentis Commonly Attributed to Achard of Saint Victor, ibidem, XXII (1960), pp. 148-191.

19. IDEM, The Commentaries on Boethius by Gilbert of Poitiers (StT, 13), Toronto, 1966, XVI-437 p.p.

20. IDEM, The Liber de Differentia naturae et personae by Hugh Etberian and the Letters Addressed to bim by Peter of Vienna and Hugh of Honau, dans MSt, XXIV (1962), pp. 1-34.

21. IDEM, Hugh of Honau and the «Liber de ignorantia», ibidem, XXV (1963), pp. 209-230. 
(ff. 394v-395v) ${ }^{22}$; de N.M. Haring encore, dix textes de commentaires sur Boèce par Thierry de Chartres et des membres de son école ${ }^{23}$, cinq oeuvres de Clarembaud d'Arras, l'un des mâ̂tres de l'Ecole de Chartres ${ }^{24}$, le Liber de dulia et latria d'un certain maître Michel, notaire à la cour papale ${ }^{25} ;$ - de P. Damon, le Preconium Augustini de Godefroid de Saint-Victor ${ }^{26}$; - de E. A. Synan, la traduction en anglais du Fons pbilosopbiae du même Godefroid ${ }^{27}$; - de M.L. Colker, l'anonyme De nobilitate animi, un produit de l'humanisme scolastique du XIII siècle ${ }^{28}$; - de E.A. Synan, le texte de dix-neuf opinions «moins acceptables» de Pierre Lombard copiées par un scribe dans le ms Bristish Museum Harley $3234^{29}$; - de W.H. Principe, certains textes de la Summa aurea de Guillaume d'Auxerre et du IIIe livre $\mathrm{du}$ Commentaire sur les Sentences de Hugues de Saint-Cher ${ }^{\mathbf{3 0}}$; - de R. J. Long, le Prologue du Commentaire sur les Sentences de Richard Fishacre ${ }^{31}$; — de C. Stroick, le De anima d'Albert

22. IDEM, In Search of Adbemar's Patristic Cellection, ibidem, XXVIII (1966), pp. 336-346.

23. IDEM, The Commentaries on Boethius by Thierry of Chartres and His Scbool (StT, 20), Toronto, 1971, 619 pp.

24. IDEM, Life and Works of Clarembald of Arras, a Twelfth Century Master of the School of Chartres (StT, 10), Toronto, 1965, XIII-276 pp.

25. IDEM, «Liber de dulia et latria» of Master Michael, Papal Notary, dans MSt, XXXIII (1971), pp. 188-200.

26. Phillip DAMON, The Preconium Augustini of Godfrey of St. Victor, dans MSt, XXII (1960), pp. 92-107.

27. GODFREY OF SAINT VICTOR, The Fountain of Pbilosopby, a Translation of Fons pbilosopbiae by E. A. SYNAN, Toronto, 1972, 89 pp.

28. Marvin L. COLKER, «De nobilitate animi», dans MSt, XXIII (1961), pp. 47-79.

29. Edward A. SYNAN, Nineteen Less Probable Opinions of Peter Lombard, dans MSt, XXVII (1965), pp. 340-343.

30. Walter H. PRINCIPE, The Theology of the Hypostatic Union in the Early Thirteenth Century. I : William of Auxtrre's Theology of the Hypostatic Union (StT, 7), 332 pp. ; II : Alexander of Hales' Theology of the Hypostatic Union (StT, 12), 254 pp. ; III : Hugh of Saint-Cher's Theology of the Hypostatic Union (StT, 19), 265 pp., Toronto, 1963, 1967, 1970. Voir encore du même The Theology of the Hypostatic Union, dans MSt, XXIV (1962), pp. 392 ss. et Hugh of Saint-Cher's Stockholm «Gloss on the Sentences»: An Abridgment ratber than a First Redaction, ibidem, XXV (1963,), pp. 372-376.

31. R. James LONG, The Science of Theology according to Richard Fishacre: Edition of the Prologue to His Comimentary on the Sentences, dans MSt, XXXIV (1972), pp. 71-98. 
le Grand dans la nouvelle édition de ses Opera omnia ${ }^{32} ;$ - de J. A. Weisheipl, les Problemata determinata XLIII attribués au même Albert le Grand ${ }^{33}$; de l'équipe de la sous-commission léonine d'Ottawa, le texte critique de l'Expositio super Job ad litteram dans l'édition léonine des Opera omnia de saint Thomas ${ }^{34}$; - de J. H. Robb, le texte nouvellement établi de la question disputée $D e$ anima de l'Aquinate ${ }^{35} ;-$ de A.A. Maurer, les traductions anglaises revues et corrigées du De ente et essentia et du In Boeth. de Trin., qu. 5 et 6 du même ${ }^{36}$; - de D. E. Dubrule, une question sur l'infinité divine de Gérard d'Abbeville $^{37}$; - de S. Martin, le texte critique des Quaestiones de cognitione animae separatae a corpore de Bernard de Trilia ${ }^{38}$; - de B. Braswell, un abrégé par Godefroid de Fontaines des questions 1-4 sur le premier livre des Topiques de Boèce de Dacie $^{39}$; - de J. Watt, la traduction anglaise du De potestate regia et papali de Jean de Paris ${ }^{40}$; de W. Dunphy, des textes de

32. ALBERT MAGNI Opera amnia, T. VII, P. I : De anima edidit Clemens STROICK, O.M.I., Monasterii Westfalorum in aedibus Aschendorff, 1968, XX-286 pp.

33. James A. WEISHEIPL, O.P., The Problemata Determinata XIIII Ascribed to Albertus Magnus (1271), dans MSt, XXII (1960), pp. 303-354.

34. SANCTI THOMAE DE AQUINO Opera omnia, T. XXVI : Expositio super Job ad litteram, Romae, ad Sanctae Sabinae, 1965, 490 pp.

35. James H. ROBB, St. Thomas Aquinas' Quaestiones de anima, A \& Newly Established » Edition of the Latin Text with an Introduction and Notes (StT, 14), Toronto, 1968, 282 pp.

36. THOMAS AQUINAS, On Being and Essence, Translated with Introduction and Notes by A. A. MAURER, 2nd revised edition, Toronto, 1968, 79 pp.; THOMAS AQUINAS, On the Division and Metbods of the Sciences, Translation of Questions Five and Six of the Commentary on the De Trinitate of Boetbius with an Introduction and Notes by A.A. MAURER, new revised edition, Totonto, 1963, XL-104 pp.

37. Diane E. DUBRULE, Gerard of Abbeville, Quodlibet XIII, Question 10, dans MSt, XXXII (1970), pp. 128-137.

38. BERNARDI TRILIAE Quaestiones de cognitione animae separatae a corpore, A Critical Edition of the Latin Text with an Introduction and Notes by Stuart MARTIN (StT, 11), Toronto, 1965, XX-427 pp.

39. Bruce BRASWELL, Godfrey of Fontaine's Abridgement of Boetius of Dacia's «Quaestiones supra librum Topicorum Aristotelis》, dans MSt, XXVI (1964), pp. 302-314.

40. JOHN OF PARIS, On Royal and Papal Power, Translated with an Introduction by John WATTS, Toronto, 1971, $261 \mathrm{pp}$. 
Pierre d'Auvergne sur la causalité efficiente ${ }^{41}$; de T. Yohoyama, le texte Sophisma: Universale est intentio de Simon de Faversham $^{42}$; - de E. A. Synan, le texte de vingt questions disputées sur les Premiers analytiques par Richard de Campsall ${ }^{43}$, le texte d'un commentaire sur les Catégories ${ }^{44}$ et d'une question sur les Premiers analytiques de Peter Bradley ${ }^{45}$, les textes enfin de l'Introitus ad Sententias et les Insolubilia de Roger Nottingham $^{46}$; - de A. Maurer, les questions sur les idées divines de Henri de Harclay ${ }^{47}$; - de E. A. Synan, le texte de quatre questions d'Adam Burley sur le Liber sex principionum ${ }^{48}$; - de $\mathrm{H}$. Shapiro et F. Scott, le texte du De deffinitione de Walter Burley, et de M. J. Kitchel, celui du De potentiis animae du même Burley ${ }^{49}$; - de P. V. Spade, les traités sur les propositions modales et hypothétiques de Richard Lavenham ${ }^{50}$; - enfin, de L. A.

41. William DUNPHY, Two Texts of Peter of Auvergne on a Twofold Efficient Cause, dans MSt, XXVI (1964), pp. 287-301; IDEM, Peter of Awvergne and the Twofold Efficient Cause, ibidem, XXVIII (1966), pp. 1.21.

42. Tetsuo YOHOYAMA, Simon of Faversham's Sopbisma: «Universale est intentio », dans MSt, XXXI (1969), pp. 1-14; Jan PINBORG, Simon of Faversham's Sopbisma: Universale est intentio, A. Supplementary Note, ibidem, XXXIII (1971), pp. 360-364.

43. Elward A. SYNAN, Works of Ricbard of Campsall. Vol. I : The Text of Twenty Disputed Questions on the Prior Analytics presented at Oxford ca. 1305 (StT, 17), Toronto, 1968, 326 pp.; IDEM, Ricbard of Campsall's First Question on the "Prior Analytics》, dans MSt, XXIII (1961), pp. $305-323$.

44. IDEM, Master Peter Bradley on the 'Categories', ibid., XXIX (1967), pp. $273-327$.

45. IDEM, $A$ Question by Peter Bradley on the «Prior Analytics》, ibidem, XXX (1968), pp. 1-21.

46. IDEM, The «Introitus ad sententias 》 of Roger Nottingham, O.F.M., ibidem, XXV (1963) , pp. 259.279 ; IDEM, The 《Insolubilia» of Roger Nottingham O.F.M., ibidem, XXVI (1964), pp. 257-270.

47. Armand MAURER, O.S.B., Henry of Harclay's Questions on the Divine Ideas, dans MSt, XXIII (1961), pp. 163-193.

48. Edward A. SYNAN, Four Questions by Adam Burley on the «Liber ser principiorum 》, dans MSt, XXXII (1970), pp. 60-90.

49. Herman SHAPIRO et Frederik SCOTT, Walter Burley's Text : De deffinitione, dans MSt, XXVII (1965), pp. 337-339; M. Jean KITCHEL, The De potentiis animae of Walter Burley, ibidem, XXXIII (1971), pp. 85-113.

50. Paul Vincent SPADE, The Treatises on Modal Propositions and on Hypothetical Proposition by Ricbard Lavenbam, dans MSt, XXXV (1973), pp. 49-59. 
Kennedy, R.E. Arnold, A. C. Millward, la traduction en anglais du traité universel de Nicolas d'Autrecourt ${ }^{51}$.

ETUDES. - Passant aux études relatives à la pensée philosophique médiévale, on constate que celles consacrées à des points de doctrine chez tel ou tel maitre l'emportent largement sur celles où l'on s'occupe de thèmes, et parmi les premières, les travaux sur saint Thomas ont largement prédominé.

THÈMES PARTICULIERS. — Dans ce domaine, les arts libéraux tels qu'entendus tout au long du moyen âge ont provoqué les études les plus nombreuses et les plus importantes. De fait, Arts libéraux et philosopbie au moyen âge fut le thème choisi pour les assises du IVe Congrès international de philosophie médiévale tenu à l'Université de Montréal du 27 août au 3 septembre 1967 et ce fut sous le même titre qu'en parurent les Actes en 1969 en un volume de XXIV-1256 pages ${ }^{52}$. Un simple examen de la table des manières révèle quelle fut la préoccupation principale des organisateurs du Congrès, à savoir, de faire présenter dans les séances plénières une série de rapports dont l'ensemble ferait voir l'histoire et l'évolution de l'enseignement des arts libéraux à partir de l'antiquité où ils avaient pris naissance jusqu'à la fin du moyen âge, puis de permettre aux chercheurs de présenter en séances de sections des communications sur des points particuliers relatifs aux arts libéraux dans leur ensemble ou à l'un ou l'autre des arts particuliers du Trivium: grammaire, rhétorique, dialectique, et du Quadrivium: arithmétique, géométrie, musique, astronomie. S'ajoutaient à ces présentations deux conférences accompagnées de projections, l'une sur l'iconographie médiévale des arts libéraux, l'autre sur la danse religieuse au moyen âge, et des rapports de commissions spéciales de travail sur les sources de la pensée médiévale, les éditions de textes médiévaux, et les rapports entre la pensée arabe et la pensée chrétienne au moyen âge. La publication de tous ces rapports, conférences, communications donne au volume de ces Actes un caractère presque encyclopédique et

51. The Universal Treatise of Nicholas of Autrecourt, Translators L. A. KENNEDY, R. E. ARNOLD, A. C. MILLWARD, Milwaukee, 1971, $165 \mathrm{pp}$.

52. Arts libéraux et pbilosopbie au moyen âge (Actes du IVe Congrès internationale de pbilosopbie médiévale), Montréal-Paris, 1969, xxiv-1256 pp., $39 \mathrm{pl}$. 
ce n'est pas trop conseiller aux bibliothèques sérieuses que d'avoir sur leurs rayons cette très riche contribution à la connaissance de la pensée médiévale. On a indiqué ici-bas quelle a été en conférences et communications la contribution canadienne à ces Actes $^{53}$. - Le thème même des arts libéraux a suscité un certain nombre d'autres travaux et recherches qui ajoutent à notre connaissance à leur sujet; ainsi pour les travaux de J.A. Weisheipl sur la classification des sciences dans la pensée médiévale et sur les programmes d'études en vigueut à la Faculté des arts d'Oxford au cours des premières années du XIVe siècle ${ }^{54}$, de J.J. Murphy sur les arts de la communication orale et écrite de 1050 à $1400^{55}$, de R.J. Schoeck sur la rhétorique à Oxford au XIVe siècle ${ }^{56}$, de

53. Voici quelle fut, en conférences et communications, la contribution canadienne au Congrès et à ses Actes: Henri-Irénée MARROU, Les arts libéraux dans l'antiquité classique, pp. 5-27; J. Reginald O'DONNELL, The Liberal Arts in the Twelfth Century with Special Reference to Alexander Nequam (1157-1217), pp. 127-135; Philippe DELHAYE, La place des arts libéraux dans les programmes scolaires du XIIle siècle, pp. 161-173; James WEISHEIPL, The Place of the Liberal Arts in the University Curriculum during the XIVth and XVth Centuries, pp. 209-213; Etienne GILSON, La philosopbie et les arts libéraux, pp. 269-271; Jean-Charles FALARDEAU, Perspectives d'un bumanisme contemporain, pp. 273-278; Louis-M. RÉGIS, L'être du langage et l'bumanisme médiéval et contemporain, pp. 281-194; Philippe VERDIER, L'iconograpbie médiévale des arts libéraux, pp. 305-354; Guy-H. ALLARD, Arts libéraus et langage choz saint Augustin, pp. 481-492; Robert Darwin CROUSE, Honorius Augustodunensis: The Arts as via ad patriam, pp. 531-539; Brian P. HENDLEY, John of Salisbury's Defense of the trivium, pp. 753 ; John A. TRENTMAN, Vincent Ferrer and His Fourteenth-Century Predecessors on a Problem of Intentionality, pp. 949-956; Jean GAGNÉ, Du Quadrivium aux scientiae mediae, pp. 975-986. - Mentionnons ici les deux articles suivants parus après la tenue du Congtès: Nicholas M. HARING, The Liberal Arts in the Sermons of Garnier of Rochefort, dans MSt, XXX (1968), pp. 47.77; James J. SHERIDAN, The Seven Liberal Arts in Alan of Lille and Peter of Compostella, dans MSt, XXV (1973), pp. 27-37.

54. James A. WEISHEIPL, Classification of the Sciences in Medieval Thought, dans MSt, XXVII (1965), pp. 54-90 ; IDEM, Curriculum of the Faculty of Arts at Oxford in the Early Fourteenth Century, ibid., XXVI (1964), pp. 143.185; IDEM, Developments in the Arts Curriculum at Oxford in the Early Fourteenth Century, ibid., XXVIII (1966), pp. 151-175.

55. James J. MURPHY, The Arts of Discourse, 1050-1400, dans MSt, XXIII (1961) , pp. 194-205.

56. R. J. SCHOECK, On Rbetoric in Fourteentb Century Oxford, dans MSt. XXX (1966), pp. 214.225. 
A. P. Campbell sur l'Ars dictaminis chez Guido Faba ${ }^{57}$, et de J. Trentman sur l'existence ou l'absence de la distinction entre langage ordinaire et langage extraordinaire en logique médiévale 58 .

Un certain nombre d'études sur d'autres thèmes sont à signaler dont quelques-unes présentent un intéressant caractère d'originalité, celle, par exemple, de L. Eldredge où l'auteur cherche à discerner la présence d'influences philosophiques dans le poème The Parlement of Foules de Chaucer ${ }^{59}$, celle de M.-D. Chenu qui, avec l'aptitude qu'on lui connaît à brosser de grands tableaux, fait voir que « l'éveil de la conscience - dans la civilisation médiévale dont nous sommes les héritiers jusque dans nos plus actuels problèmes - fut l'effet de cette admirable convergence des phénomènes de civilisation, dans de hauts esprits et de spirituels amoureux qui le firent advenir dans une très grande histoire» (p. 80) ${ }^{60}$, celle de L. Valcke qui a tenté d'esquisser à grands traits la mentalité «préphilosophique » qui régna à l'époque du moyen âge à son déclin ${ }^{61}$, celle de $\mathrm{R}$. de Roover qui, à l'encontre des économistes modernes, affirme l'existence d'une pensée économique médiévale et montre que les scolastiques en ce domaine ont été avant tout des théoriciens préoccupés de formuler des principes plutôt que d'en appliquer aux situations concrètes ${ }^{62}$. Mentionnons enfin l'ouvrage de première importance que nous a présenté $\mathrm{C}$. Bérubé qui a fait l'histoire de la connaissance de l'individuel au moyen âge, problème qui n'en est nullement un de détail, mais qui se trouve plutôt au point de convergence de la métaphysique et de la psychologie et qui a des répercussions immédiates en critique et en théologie, comme le

57. A. P. CAMPBELL, The Perfection of Ars Dictaminis in Guido Faba, dans RUO, 39, 2 (1969), pp. 315-321.

58. John TRENTMAN, Extraordinary Language and Medieval Logic, dans Dialogue, VII, 2 (1968), pp. 286-291.

59. Laurence ELDREDGE, Poetry and Pbilosopby in 8 The Parlement of Foules $\gg$, dans RUO, 40, 3 (1970), pp. 441-459.

60. M.-D. CHENU, O.P., L'éveil de la conscience dans la civilisation médiévale (CAG 1968), Montréal-Paris, 1969, 84 pp.

61. Louis VALCKE, Le nominalisme et le déclin du Moyen âge, dans Dialogue, III, (1964), Pp. 248-261.

62. R. DE ROOVER, La pensée économique des scolastiques. Doctrines et métbodes (CAG 1970), Montréal-Paris, 1971, 108 pp. 
rappelle l'auteur (p. 278) ${ }^{63}$. Celui-ci, comme la critique l'a reconnu, a réalisé un excellent travail qui, pour n'être pas complet (par exemple, les maîtres de la faculté des arts n'y sont pas étudiés), n'en démêle pas moins la question pour ce qui regarde les positions des théologiens. Le même sujet avait déjà tenté un autre auteur qui a malheureusement appliqué une méthode qu'on se doit de recommander de ne pas imiter ${ }^{64}$.

LES MAÎTRES. - Arabes et orientaux. La pensée arabe et orientale a attiré l'attention de quelques travailleurs. Ainsi M. E. Marmura et J. M. Rist ont examiné le problème de l'existence et de l'unité divines tel que présenté et discuté par Al-Kindi ${ }^{65}$; M. E. Marmura encore, celui du nombre infini des âmes d'après Avicenne ${ }^{66}$, G. Jalbert, celui du nécessaire et du possible chez Avicenne ${ }^{67}$, de la nécessité et de la contingence chez Aristote et Averroès ${ }^{68}, \mathrm{M}$. Fakhry, celui du rapport entre la philosophie et l'écriture dans la théologie du Commentator ${ }^{69}$. R. Lemay a, pour sa part, établi qu'une partie de la physique d'Aristote a été retrouvée dans le monde latin à travers l'astrologie arabe, en particulier à travers l'Introductorium in astronomiam d'Abu Ma'shar ${ }^{70}$. A signaler, enfin, à cet endroit, l'inventaire que M. DeDurand a présenté du grand Livre des Questions de Grégorie Tatev. Les rapprochements qu'on peut faire de cette somme arménienne du XIVe siècle quant à son contenu, ses sources, et son

63. Camille BÉRUBÉ, O.F.M. Cap., La connaissance de l'individuel au moyen âge, Montréal-Paris, 1964, XII-316 pp.

64. Emile BOUCHER, Investigatio historica supra cognitionem rerum materialium singularium existentium, dans SMR, IV (1961), pp. 99-126; 185-228.

65. Michael E. MARMURA and John M. RIST, Al-Kindi's Discussion of Divine Existence and Oneness, dans MSt, XXV (1963), pp. 338-354.

66. Michael E. MARMURA, Avicenna and the Problem of the Infinite Number Souls, dans MSt, XXIII (1960), pp. 232-239.

67. Guy JALBERT, O.M.I., Le nécessaire et le possible dans la pbilosopbie d'Avicenne, dans RUO, 30, 2 (1960), pp. 89*-101*.

68. IDEM, La nécessité et la contingence chez Aristote et Averroès, dans RUO, 30,1 (1960) , pp. $21 *-36 *$.

69. Majid FAKHRY, Pbilosopby and Scripture in the Theology of Averroes, dans MSt, XXX (1968), pp. 78-89.

70. R. LEMAY, Abu Ma' shar and Latin Aristotelianism in the Twelfth Century. The Recovery of Aristotle's Natural Pbilosopby through Arabic Astro. $\operatorname{logy}$ (American University of Beirut, Publication of the Faculty of Arts and Sciences, Oriental Series, 38), Beirut, 1962, XL-468 pp. 
genre littéraire avec les Sommes de l'Occident latin sont des plus intéressants et instructifs ${ }^{71}$.

Maîtres du XIIe siècle. - Des maîtres antérieurs au XIle siècle, c'est Jean Scot Erigène qui attire le plus souvent l'attention des chercheurs et de fait une étude lui a été consacrée par B. Stock qui, en parcourant pas à pas le Periphyseon ou $D e$ divisione naturae de Jean Scot dans une optique d'anthropologie philosophique, a dégagé la conception de celui-ci sur l'homme idéal et sur ses rapports avec l'histoire et le temps ${ }^{72}$. - De Jean Scot on passe directement aux maîtres du XIIe siècle avec Anselme de Cantorbéry en premier lieu. Son Cur Deus Homo a fait l'objet d'une solide analyse de la part de Th.-A. Audet. Cette analyse fait ressortir la maîtrise dialectique d'Anselme, mais l'introduction qui la précède pourrait servir d'introduction à l'étude de saint Anselme lui-même, le Cur Deus Homo y étant situé par rapport aux autres oeuvres anselmiennes et dans la perspective des autres études historiques qui lui ont été consacrées ${ }^{73}$. A. C. Pegis a, de son côté, étudié l'argument du Proslogion. Il en conclut que cet argument ne doit pas être interprété dans le contexte de la problématique et de la pensée du XIIIe siècle, mais bien pour ce qu'il est, un argument qui procède de la présence cachée de Dieu dans l'esprit d'Anselme à l'affirmation nécessaire de son existence transcendante et ineffable ${ }^{74}$. - Plusieurs maittres de l'Ecole de Chartres ont retenu l'attention. N.M. Haring a établi que Thierry de Chartres, dans son commentaire sur le De inventione de Cicéron, a copié toute la partie sur la rhétorique du $D e$ divisione philosopbiae de Dominique Gundissalinus ${ }^{75}$; T. Silverstein a

71. Mathieu DEDURAND, O.P., Une somme arménienne au XIVe siècle dans Etudes d'bistoire littéraire et doctrinale, 4e série (PIEM, XIX), MontréalParis, 1968, pp. 217-277.

72. B. STOCK, The Philosophical Anthropology of Johannes Scottus Eriugena, dans Studi Medievali, 3e série, 8 (1967), pp. 1.57.

73. Th.-A. AUDET, O.P., Problématique et structure du Cur Deus Homo, dans Etudes d'bistoire littéraire et doctrinale, 4e série (PIEM, XIX), MontréalParis, 1968, pp. 7-115.

74. Anton C. PEGIS, St. Anselm and the Argument of the Proslogion, dans MSt, XXVIII (1966), pp. 228-267.

75. N. HARING, Tbierry of Chartres and Dominicus Gundissalinus, dans MSt, XXVI (1964), pp. 271-286. 
étudié l'origine des mots bomiomeria et organica utilisés par Guillaume de Conches dans le contexte d'une explication des éléments ${ }^{76}$; J. R. O'Donnell établit que le Commentaire sur l'Evieide de Bernard Silvestre est une sorte d'encyclopédie tentant de mettre les lettres classiques au service des gens de son temps ${ }^{77}$, alors que B. Stock montre que dans sa Cosmographia le même Bernard a une vision du monde essentiellement empirique d'où le mythe n'a pas été éliminé, ou, ce qui est plus probable, qu’il a une vision mythique du monde à laquelle est venu s'ajouter un intérêt naissant pour le monde réel ${ }^{78}$; E. K. Tolan, enfin, tente de montrer que les Metalogicon et Policraticus de Jean de Salisbury sont des oeuvres où certaines tendances de l'humanisme médiéval sont examinées et évaluées et que, de fait, l'humanisme de Jean se tient à l'intérieur de frontières volontairement restreintes ${ }^{79}$. - En ce qui concerne l'école porrétaine, en plus des éditions de textes indiquées déjà et dues en majeure partie aux efforts infatigables de N. M. Haring, il y a à mentionner une ćtude de ce dernier sur l'utilisation des Pères grecs par Gilbert lui-même ainsi que par les membres de son école ${ }^{80}$, une autre sur les emprunts faits à Gilbert par Simon de Tournai dans ses Sentences ${ }^{81}$, celle enfin consacrée par A. Dondaine aux écrits de la « petite école » porrétaine, étant compris sous cette expression Adhémar de Saint-Ruf, Hugues de Honau, Pierre de Vienne et

76. Theodore SILVERSTEIN, Guillaume de Conches and the Elements: Homiomeria and Organica, in MSt, XXVI (1964), pp. 363-367.

75. N. HARING, Thierry of Chartres and Dominicus Gundissalinus, dans MSt, XXVI (1964), pp. 271-286.

76. Theodore SILVERSTEIN, Guillaume de Conches and the Elements: Homiomeria and Organica, dans MSt, XXVI (1964), pp. 363-367.

77. J. Reginald O'DONNELL, C.S.B., The Sources and Meaning of Bernard Silvester's Commentary on the Aeneid, dans MSt, XXIV (1962), pp. 233-249.

78. Brian STOCK, Myth and Science in the Twelfth Century: A Study of Bernard Silvester, Princeton (N.J.) , 1972, XVI-331 pp.

79. E. K. TOLAN, Jobn of Salisbury and the Problem of Medieval Humanism, dans Etudes d'bistoire littéraire et doctrinale, 4e série (PIEM, XIX), Montréal-Paris, 1968, pp. 189-199. Sur Jean de Salisbury, voir encore l'article de B.P. HENDLEY, mentionné à la note 53 .

80. Nicholas M. HARING, The Porretans and the Greek Fathers, dans MSt, XXIV (1962), pp. 181-209.

81. IDEM, Simon of Tournai and Gilbert of Poitiers, dans MSt, XXVII (1965) , pp. 325.330. 
Hugues Ethérien ${ }^{82}$. - Pierre Lombard : à celui-ci dont les quatre livres des Sentences ont été le manuel scolaire utilisé dans les facultés de théologie pendant quatre siècles, $\mathrm{Ph}$. Delhaye a consacré, à l'occasion du huitième centenaire de sa mort, une intéressante conférence dans laquelle, après avoir fait un tour d'horizon de la matière morale telle que vue et traitée par le Lombard, il fait voir que celui-ci a développé une morale à la fois doctrinale et axée sur les valeurs positives ${ }^{83}$.

Maîtres des XIIIe-XIVe siècles. - Maîtres franciscains. Le Sapientiale de Thomas d'York a été soumis par E. Scully à un examen d'où se dégage la conclusion que la tradition augustinienne a été dotée chez les premiers franciscains d'une métaphysique à la fois nouvelle et libérale, tirée principalement d'Aristote et des grands penseurs juifs et arabes du moyen âge ${ }^{84}$. C'est surtout saint Bonaventure, cependant, qui a attiré l'attention des chercheurs. Au chapitre des sources, C. Bérubé a montré que Guibert de Tournai, O.F.M. et Robert Grosseteste ont été les premières sources, jusqu'ici inconnues, des doctrines de l'illumination et de la connaissance de Dieu chez Bonaventure, et avec l'aide de S. Gieben il a publié les trois chapitres les plus significatifs sur le sujet du Rudimentum doctrinae de Guibert ${ }^{85}$; au plan de la chronologie, J. T. Quinn a repris le problème des dates de la vie de saint Bonaventure pour les années $1217-1257^{86}$; au plan de la technique, G.-H. Allard a étudié la nature, le fonctionnement, les limites et les aires d'applications de la reductio bonaventurienne en y ajoutant quelques remarques sur la resolutio ${ }^{87}$; au plan

82. Antoine DONDAINE, O.P., Écrits de la «petite école» porrétaine (CAG 1962), Montréal-Paris, $1962,68 \mathrm{pp}$.

83. Philippe DELHAYE, Pierre Lombard: sa vie, ses oeuvres, sa morale (CAG 1960), Montréal-Paris, 1961, 112 pp.

84. Edgar SCULLY, The Power of Physical Bodies according to Thomas of York, dans ScE, XIV (1962), pp. 109-134.

85. Camille BÉRUBÉ et Servus GIEBEN, O.F.M. Cap. Guibert de Tournai et Robert Grosseteste sources inconnues de saint Bonaventure, suivi de l'édition critique de trois chapitres $d u$ Rudimentum Doctrinae de Guibert de Tournai, dans S. Bonaventure 1274-1974, t. II, Grottaferrata (Rome), pp. 627.654 .

86. John F. QUINN, Chronology of St. Bonaventure (1217-1257), dans Franciscan Studies, 32 (1972), pp. 168-186.

87. Guy-H. ALIARD, La technique de la «Reductio» chez Bonaventure, dans S. Bonaventura 1274-1974, t. II, Grottaferrata (Roma), pp. 395-416. 
des cheminements doctrinaux, $\mathrm{C}$. Bérubé s'est arrêté au rôle que Bonaventure attribue à la philosophie dans la promotion de la sagesse, de même qu'aux rapports entre la philosophie et les autres habitus intellectuels qui constituent la sagesse chrétienne en son intégrité ${ }^{88}$, alors que A. C. Pegis a montré que c'est volontairement que Bonaventure a choisi de vivre dans un univers augustinien plutôt que dans l'univers physique d'Aristote, et de chercher à y découvrir le chemin qui mène à Dieu par la voie illuminative déjà suivie par Augustin et Anselme ${ }^{89}$, qu'il ne s'est pas soucié de s'assimiler la science aristotélicienne pour elle-même, mais qu'il a vécu de préférence, en tant que théologien, à la lumière de l'Ecriture dans l'amour de Dieu ${ }^{90}$. - A signaler au sujet des maîtres franciscains de la fin du XIIIe et du début du XIVe siècle, les contributions de B. Martel sur la psychologie de Gonsalve d'Espagne dont il examine la pensée sur les structures du composé humain et sur l'activité des puissances de l'âme et chez qui il retrouve les doctrines franciscaines traditionnelles de la multiplicité des formes et de la priorité de la volonté sur l'intelligence ${ }^{91}$, de C. Bérubé sur l'histoire des preuves de l'existence de Dieu chez Duns Scot dans laquelle il émet l'hypothèse d'une évolution du docteur subtil qui serait «parti d'une méthode où l'apriorisme anselmien centré sur les perfections absolues de Dieu s'allie à un certain réalisme avicennien des natures, pour évoluer vers des positions où l'apostériorisme soi-disant aristotélico-thomiste aurait finalement un rôle déterminant $\gg$ (p. 17) ${ }^{92}$, de P. Vignaux sur l'être comme perfection chez François de Meyronnes ${ }^{93}$, de A.

88. C. BÉRUBÉ, De la pbilosopbie à la sagesse dans l'itinéraire bonaventurien, dans Collectanea franciscana, 38 (1968), pp. 257-307.

89. Anton C. PEGIS, The Bonaventurean Way to God, dans MSt, XXIX (1967), pp. 206-242.

90. Anton C. PEGIS, St. Bonaventure Revisited, dans S. Bonaventura 1274-1974. II - Studia de vita, mente, fontibus et operibus sancti Bonaventurae, Grottaferrata (Roma), 1973, pp. 21-44.

91. Benoît MARTEL, O.F.M. Cap., La psychologie de Gonsalve d'Espagne (PIEM, XXI), Montréal-Patis, 1969, 208 pp.

92. Camille BÉRUBÉ, O.F.M. Cap., Pour une bistoire des prewves de l'exis. tence de Dieu chez Duns Scot, dans Deus et Homo ad mentem J. Duns Scoti (Studia scbolasticoscotistica, 5), Rome, 1972, pp. 17-46.

93. P. VIGNAUX, L'être comme perfection selon Francois de Meyronnes, dans Etudes d'bistoire littéraire et doctrinale, 3e série (PIEM, XVII), MontréalParis, 1962, pp. 219-318. 
Maurer et A. Caird, enfin, sur le rôle de l'infini dans la pensée du même François de Meyronnes ${ }^{94}$.

Maîtres dominicains. Deux études sur Albert le Grand: dans la première, F. J. Catania examine un ensemble de textes du commentaire d'Albert sur les Sentences du Lombard pour découvrir si, chez lui, l'infini de Dieu fait partie de son être même ou s'il doit être considéré comme extrinsèque à cet être; Albert est rangé dans la catégorie des penseurs qui ont adopté la deuxième position ${ }^{95}$; dans la seconde, L.-B. Geiger a voulu mettre en lumière l'existence chez Albert d'une conception générale où fusionnent la thèse de la vie, acte de l'âme, et celle de l'esse, acte de l'essence, position métaphysique au sujet de laquelle il y aurait intérêt à se demander si elle est l'oeuvre des penseurs du moyen âge ou simplement un emprunt à leurs prédécesseurs grecs ou arabes ${ }^{96}$. - Thomas d'Aquin. Comme on le mentionnait plus haut, c'est la pensée de Thomas d'Aquin qui a fait l'objet du plus grand nombre d'études. On pourrait presque dire que c'est tout le spectrum philosophique qui y a passé, soit en monographies, soit en articles. Nous devrons nous limiter à dire un mot des plus importants, quitte à simplement signaler les autres dans une sèche énumération. Comme instruments pour aborder et comprendre les écrits et la pensée de saint Thomas, deux contributions importantes: l'une est la présentation en version anglaise avec mise à jour bibliographique de l'Introduction à l'étude de saint Thomas d'Aquin de M.-D. Chenu dont la critique a abondamment reconnu la valeur et les mérites dès sa parution pour la première fois en $1950^{97}$; l'autre est l'ouvrage tout récent de J.A. Weisheipl sur la vie, la pensée et les oeuvres de saint Thomas qui est replacé dans son milieu social, politique et culturel et dont l'évo-

94. Armand MAURER and Alfred P. CAIRD, The Role of Infinity in the Tbought of Francis of Meyronnes, dans MSt, XXXIII (1971), pp. 201-227.

95. Francis J. CATANIA, Divine Infinity in Albert the Great's Commentary on the Sentences of Peter Lombard, dans MSt, XXII (1960), pp. 27-42.

96. L.-B. GEIGER, O.P., La vie, acte essentiel de l'âme - l'esse, acte de l'essence d'après Albert le Grand, dans Etudes d'bistoire littéraire et doctrinale, 3e série (PIEM, XVII), Montréal-Paris, 1962, pp. 49-116.

97. M.-D. CHENU, O.P., Toward Understanding Saint Thomas, Translated by Albert-M. LANDRY, O.P. and Dominic HUGHES, O.P.. Chicago, 1964, $\mathrm{X}-386 \mathrm{pp}$. 
lution intellectuelle est retracée. S'y trouve à la fin du volume un bref catalogue des oeuvres authentiques de Thomas donnant les lieux et années de composition et un bref commentaire sur chacune des oeuvres ${ }^{98}$. Relativement à la philosophie de saint Thomas elle-même, A. C. Pegis a donné la réponse au jugement négatif porté par $\mathrm{P}$. Duhem dans Le système du monde ${ }^{99}$ et tenté de définir ce que fut, selon lui, cette philosophie du théologien Thomas dont nous ne savons pas à l'intérieur de quels cadres et structures il l'aurait présentée s'il l'avait écrite ${ }^{100}$. En métaphysique, c'est probablement à $\mathrm{J}$. Owens qu'il faut remettre la palme. Celui-ci, en effet, commençait en 1957 la publication dans $M e-$ diaeval Studies d'une série d'articles sur les problèmes fondamentaux de la métaphysique. On en verra la matière exacte dans la reproduction des titres que nous donnons en note ${ }^{101}$. On peut peut-être exprimer l'espoir que le $\mathrm{P}$. Owens voudra rendre à ses lecteurs le service de publier en un volume la synthèse de la matière de ces articles! Encore en métaphysique, la recherche entreprise sur la nécessité et la contingence par G. Jalbert a abouti

98. J. A. WEISHEIPL, O.P., Friar Thomas d'Aquino. His Life, Thought and Works, New York, 1973, 464 pp.

99. Pierre DUHEM, Le système du monde. Histoire des doctrines cosmologiques de Platon à Copernic, t. V, Paris, 1917, pp. 486-570.

100. Anton C. PEGIS, St. Thomas and Pbilosopby (The Aquinas Lecture 1964), Milwaukee, 1964, $94 \mathrm{pp}$.

101. Joseph OWENS, C.SS.R., Common Nature: A Point of Comparison between Thomistic and Scotistic Metaphysics, dans MSt, XIX (1957), pp. 1-14; The Accidental and Essential Character of Being in the Doctrine of St. Thomas Aquinas, ibid., XX (1958), pp. 1-40; Thomistic Common Nature and Platonic Idea, ibid., XXI (1959), pp. 211-223; Diversity and Community of Being in St. Thomas Aquinas, ibid., XXII (1960), pp. 257-302; Unity and Essence in St. Thomas Aquinas, ibid., XXIII (1961), pp. 240-259; Analogy as a Thomistic Approach to Being, ibid., XXIV (1962), pp. 303-322; The Unity in a Thomistic Pbilosopby of Man, ibid., XXV (1963), pp. 54-82; The «Analytics» and Tbomistic Metapbysical Procedure, ibid., XXVI (1964), pp. 83-108; Quiddity and Real Distinction in St. Thomas Aquinas, ibid., XXVII (1965), pp. 1-22; Aquinas and the Proof from the Pbysics, ibid., XXVIII, (1966), pp. 119-150; Actuality in the "Prima Via》 of St. Tbomas, ibid., XXIX (1967), pp. 26-46; 'Cause of Necessity' in Aquinas' Tertia Via, ibid.. XXXIII (1971), pp. 21-45; Immobility and Existence for Aquinas, ibid.. XXX (1968), pp. 22-46; Aquinas - Existential Permanence and Flux, ibid., XXXI (1969), pp. 71-92; Judgment and Truth in Aquinas, ibid., XXXII (1970), pp. 138-158; Metaphysical Separation in Aquinas, ibid., XXXIV (1972), pp. 287-306. 
à un ouvrage remarqué ${ }^{102}$. Avant d'examiner le problème dans la suite chronologique des textes de saint Thomas, l'auteur passe en revue les positions d'Aristote, Avicenne, Avicébron, Averroès, Mä̈monide, Guillaume d'Auvergne, Albert le Grand et Bonaventure. L'une de ses grandes conclusions est que Thomas est passé d'une interprétation avicennienne de la question à un pur aristotélisme qu'il a prolongé et perfectionné. Les vues anthropologiques de saint Thomas ont été un autre objet de recherches importantes et valables. A. C. Pegis a tenté de dégager les facteurs qui ont été à l'origine de la conception que se fait saint Thomas de l'homme, créature intellectuelle immortelle qui pourtant incorpore en une unité parfaite l'univers de la nature et qui ne se réalise que dans un devenir ${ }^{103}$. G. Langevin, d'autre part, «se propose de retrouver, aux différentes étapes de son développement, avec ses nuances et ses méandres, la pensée de saint Thomas sur les points d'appui que la nature offre à la grâce en l'esprit créé » (p. 17) ${ }^{104}$. J. Castonguay conclut, après une recherche sur la mémoire dans la pensée ancienne et médiévale (grecs, latins, musulmans et juifs), et une étude des diverses formes de la mémoire et de la genèse de l'acte mnémonique et ses problèmes dans les textes de saint Thomas, que celui-ci, loin de présenter ce qui ne serait qu'un décalque de la pensée antérieure, a réussi une systématisation nouvelle et vraiment originale ${ }^{105}$. R. Tittley, enfin, s'est adressé aux textes de saint Thomas d'abord pour préciser la nature de la passion physique proprement dite et celle de la passion animale et ensuite établir que l'Aquinate a commencé par concevoir la douleur sensible comme sensation tactile d'une lésion corporelle pour passer plus tard à la conception de cette douleur comme passion et acte de l'appétit sensible ${ }^{106}$. En épistémologie, cinq articles de B. Lonergan portant sur le verbum chez

102. Guy JALBERT, O.M.I., Nécessité et contingence chez saint Thomas d'Aquin et ses prédécesseurs (Publications sériées de l'Université d'Ottawa, LXIV), Ottawa, 1961, $256 \mathrm{pp}$.

103. Anton C. PEGIS, At the Origins of the Thomistic Notion of Man (Saint Augustine Lecture, 1962), New York-London, 1963, VIII-82 pp.

104. Gilles LANGEVIN, S.J., "Capax Dei». La créature intellectuelle et l'intimité de Dieu (Studia, Recherches de théologie et de philosopbie publiées par les facultés s.j. de Montréal, 20), Bruges-Paris, 1966, 144 pp.

105. J. CASTONGUAY, Psychologie de la mémoire, Montréal, 1963, $262 \mathrm{pp}$.

106. Rémi TITTLEY, La douleur sensible, Montréal, 1967, V-157 pp. 
saint Thomas, parus d'abord dans Theological Studies, ont été reproduits après avoir été revus par leur auteur, une première fois en traduction française dans la Bibliothèque des arcbives de pbilosopbie, une seconde fois dans l'anglais original édités par D. B. Burrell ${ }^{107}$. Le P. Lonergan a préparé lui-même les introduction et épilogue aux volumes, y expliquant le but de ses recherches qui était de clarifier le sens du verbum dans les contextes divers où l'Aquinate en parle et la méthode qu'il a suivie pour y arriver et qu'il décrit ainsi : "C'est seulement grâce à un travail lent, qui se reprend, qui revient sans cesse sur les données, gagnant ici une petite intuition et là une autre, en suivant des fausses pistes et en profitant de beaucoup d'erreurs, en faisant de continuels ajustements et des transformations cumulatives, des suppositions, des perspectives et des concepts que l'on possède en commençant, qu'on peut espérer comprendre ce que saint Thomas a compris et ce qu'il voulait dire. C'est là la méthode que j'ai employée....» (p. 228). Le même thème a suscité l'étude de J. Chênevert, mais celui-ci a limité son enquête au Commentaire sur les Sentences et il l'élabore sous les sous-titres de "Verbum et species intellecta》 et de «Ratio verbi », pour ensuite tenter de découvrir les facteurs qui ont pu déterminer l'état initial de la pensée de saint Thomas au sujet du verbe mental ${ }^{108}$. Puis, c'est à l'étude de l'acte du jugement que B. Garceau s'est attaqué, étudiant de celui-ci le vocabulaire, les sources et la doctrine chez l'Aquinate ${ }^{109}$. Le résultat principal auquel l'aura conduit sa minutieuse enquête sera d'avoir découvert ce qu'est pour lui la note essentielle du jugement : "Cette note essentielle, ou ce noyau de signification, c'est le discernement par une vertu cognitive de son objet propre. Dans l'activité complexe du connaître, les espèces, les concepts, les

107. Bernard LONERGAN, La notion de verbe dans les écrits de saint Thomas d'Aquin (Bibliothèque des Archives de pbilosopbie, nouv. série, 5). Paris, 1967, X.260 pp.; IDEM, Verbum, Word and Idea in Aquinas, Edited by David B. BURRELL, C.S.C., London, 1968, XVIII-300 pp.

108. Jacques CHÊNEVERT, S.J., Le Verbum dans le Commentaire sur les Sentences de saint Thomas d'Aquin, dans ScE, XIII (1961), pp. 191-223; 359-390.

109. Benoit GARCEAU, O.M.I., Judicium. Vocabulaire, sources, doctrine de saint Thomas d'Aquin (PIEM, XX), Montréal-Paris, 1968, 286 pp. ; voir encore IDEM, La doctrine thomiste du jugement, dans RUO, 32. 4 (1962) , pp. $215^{*}-237^{*} ; 33$, I (1963), pp. $5^{*}-27^{*}$. 
énoncés, le discours sont, pour nous, des facteurs indispensables; mais ils n'ont de sens que parce qu'ils sont, soit l'expression, soit la condition de l'acte par lequel l'âme reconnaît ce pour quoi elle est faite: le réel dans sa valeur de vérité et de bien» (p. 254). En morale, Ch. Murin a tenté de définir ce qu'est l'ens morale lui-même, étude négligée par la plupart des auteurs qui traitent de philosophie morale. Son enquête se fait en deux temps : étude de l'ens morale par rapport aux subjecta des autres disciplines philosophiques (métaphysique, psychologie, savoirs pratiques, logique), puis examen poussé de l'être moral dans le contexte de l'être de raison. Au terme du cheminement dialectique parcouru, l'auteur pense avoir atteint son but qui était de « faire voir d'une façon indubitable ce que n'est pas l'être moral, et mettre l'esprit sur la piste qu'il faudrait longuement parcourir pour découvrir la nature intime de cette entité mystérieuse qui commande toute l'activité humaine et conditionne son bonheur éternel» ( $\mathrm{p}$. $237)^{110} . \mathrm{J}$. Tonneau, pour «comprendre pourquoi saint Thomas a pu, comme par préterition, refuser à l'obligation le premier rôle en morale et en même temps ne pas songer à nous rassurer, à s'en expliquer, à justifier ce parti » (p. 13), a examiné ce qui fait la nature de l'obligation dans l'obligation selon l'excellence, l'obligation contractuelle, l'obligation du précepte, de la loi, de la conscience; il conclut qu'une "morale de l'obligation, même vécue sous sa forme la plus parfaite qui est la morale sous l'empire de la religion ou la sainteté, suppose toujours, si on veut l'expliquer, une morale du bien dû selon l'ordre d'une nature » (p. 126) ${ }^{111}$. Mentionnons enfin, toujours en morale, la nouvelle édition, revue et corrigée, de L'bumanisme politique de saint Thomas d'Aquin. Individu et état de L. Lachance qui a fait ressortir les positions foncièrement humaines de saint Thomas dans sa doctrine si approfondie et si nuancée des relations de l'individu

110. Charles MURIN, Recherche sur la nature de l'être moral dans l'oeuvre de saint Thomas, dans Études d'bistoire littéraire et doctrinale (PIEM, XVIII), Montréal-Paris, 1962, pp. 175-237.

111. Jean TONNEAU, O.P., Obligation et absolu en morale (CAG 1964), Montréal-Paris, 1965, 127 pp.

112. Louis LACHANCE, O.P., L'bumanisme politique de saint Thomas d'Aquin, Individu et Etat, 2e édit., Paris-Montréal, 1965. 400 pp. 
et de l'état ${ }^{112}$. Au chapitre enfin des comparaisons entre l'une ou l'autre des positions de saint Thomas et celles de philosophes modernes, trois études à signaler. La première, de B. Rioux, a pour but de confronter les pensées de saint Thomas et de Heidegger sur l'être et la vérité. Au terme de son cheminement à travers la vérité du «Dasein » et de l'être chez Heidegger, à travers la vérité du jugement et celle de l'être chez Thomas, à travers sa conclusion critique dans laquelle les points forts et faibles de l'un et l'autre penseur sont signalés et confrontés, l'auteur conclut sur cette note d'une belle élévation: «...cette méditation sur l'Etre (de Heidegger) témoigne de la grandeur de l'homme en ce temps de misère. Elle rappelle l'homme à sa vocation quasi religieuse de berger de l'Etre, par-delà toute volonté de puissance s'exerçant sur les choses et les hommes. Quant à nous, elle nous a stimulé à relire Thomas d'Aquin dans une perspective plus décidément ontologique (p. 259) ${ }^{113}$. Les deux autres sont de L. Martinelli: l'une confronte l'analyse linguistique moderne à l'analyse thomiste et conclut que la seconde est en pratique constante chez Thomas comme méthode, mais qu'elle n'a pas connu le glissement insensible de la méthode vers l'objet et la transmutation de la méthode en objet; «La grammaire, la dialectique sont impuissantes à donner la vie à une philosophie, si l'esprit n'a plus de serres sur le réel. Cette conviction inébranlable est la clef des activités d'analyse linguistique dans l'oeuvre de saint Thomas d'Aquin» ( $p$. 77) ${ }^{114}$; la seconde pose la question de l'existence d'une philosophie des valeurs chez saint Thomas, le problème des valeurs étant pensé «nouveau » depuis Friedrich Nietzsche. La réponse donnée par l'auteur est catégorique : "l'oeuvre de Thomas d'Aquin présente une ontologie, une psychologie, une logique des valeurs» (p. 153), et à la justification de cette affirmation sont consacrées des pages denses qui prouvent qu'un problème dit «nouveau»

113. Bertrand RIOUX, L'être et la vérité chez Heidegger et saint Thomas d'Aquin, Montréal, 1963, XI-270 pp.; voir aussi IDEM, La notion de vérité chez Heidegger et saint Thomas d'Aquin, dans Saint Thomas d'Aquin aujourd'bui (Recherches de philosopbie, 6), Paris, 1963, pp. 197-217.

114. Lucien MARTINELLI, Thomas d'Aquin et l'analyse linguistique (CAG 1963), Montréal-Paris, $1963,79 \mathrm{pp}$.

115. IDEM, Thomisme et valeurs, dans Etudes d'bistoire littéraire et doctrinale, 3e série (PIEM, XVII), Montréal-Paris, 1962, pp. 151-174. 
peut être fort ancien dans son objet, fort ancien aussi dans la diversité des réponses qu'on a formulées à son sujet ${ }^{115}$.

En plus des quelques ouvrages que nous venons de mentionner, un très grand nombre d'autres travaux sur la pensée de saint Thomas mériteraient autre chose qu'une simple mention bibliographique, mais nous devons nous en tenir à leur sujet à l'énumération suivante qui aura au moins l'avantage de donner au lecteur une idée plus juste de la production des années qui nous occupent. Voici dans l'ordre alphabétique les thèmes sur lesquels il s'est fait de la recherche: l'abstraction ${ }^{116}$, l'admiration ${ }^{117}$, l'âme humaine ${ }^{118}$, l'analogie ${ }^{119}$, l'appréhension ${ }^{120}$, l'art ${ }^{121}$, les biens temporels ${ }^{122}$, la bénignité divine ${ }^{123}$, les quatre causes ${ }^{124}$, la certitude ${ }^{125}$, la colère ${ }^{126}$, le concept ${ }^{127}$, la connaissance ${ }^{128}$, la

116. Cornelius J. KELLY, Abstraction and Existence, dans LTP, XXI (1965), pp. 17.42 ; Charles DE KONINCK, Abstraction from Matter, I, dans LTP, XIII (1957), pp. 133-196; II, ibid., XVI (1960), pp. 53-69; ibid., XVI (1960), pp. 167-188.

117. Guy GODIN, La notion d'admiration, dans LTP, XVII (1961), pp. 35-75; L'admiration, principe de la recherche pbilosopbique, ibid., pp. 213-242.

118. James P. BLEDSOE, Aquinas on the Soul, dans LTP, XXIX (1973), pp. 273-290; Jean RACETTE, S.J., L'étude de l'âme bumaine relève-t-elle de la philosopbie de la nature, dans $S_{c E}$, XII (1960), pp. 105-117.

119. Ralph MINERNY, The Ratio Communis of the Analogous Name, dans LTP, XVIII (1962), pp. 9-34; M. GERVAIS, Note critique: L'analogie selon saint Thomas et Karl Barth dans LTP, XXIX, 2 (1973), pp. 187192 ; M. S. O'NEILL, Some Remarks on the Analogy of God and Credtures in St. Thomas Aquinas, dans MSt, XXIII (1961), pp. 206-215; voir encore notes 101 et 155 .

120. Voir plus bas, note 147.

121. SOEUR SAINT-MARTIN-DE-TOURS, Pourquoi l'art doit imiter la nature, dans LTP, XXI (1965), pp. 175-190.

122. Dalton J. FORBES, Temporal Goods in the Christian Economy, A Thomistic Syntbesis, dans RUO, 30, 4 (1960), pp. 185*-206*; 31, 1 (1961), pp. $39 *-71 *$.

123. Raymond LAFLAMME, Nature de la bénignité divine, dans LTP XIX (1963), pp. 22-48.

124. Oliva BLANCHETTE, The Four Causes as Texture of the Universe, dans LTP, XXV (1969), pp. 59-87.

125. Lorenzo ROY, La certitude en matière morale, dans LTP (1963), pp. 120-170.

126. Martin BLAIS, La colère selon Sénèque et selon saint Thomas, dans LTP, XX (1964), pp. 247-290.

127. Voir plus bas, note 146.

128. Robert L. FARICY, S.J., Connatural Knowledge, dans ScE, XVI (1964), pp. 155-163; Louis-Emile BLANCHET, Simples réflexions sur le désir de connaitre, dans LTP, XXVII (1971), pp. 7-24; Frederick E. CROWE, 
contingence ${ }^{129}$, la dépendarfe ${ }^{130}$, le droit naturel ${ }^{131}$, l'économie chrétienne $^{132}$, l'éducation ${ }^{133}$, l'erreur ${ }^{134}$, l'éthique ${ }^{135}$, l'être ${ }^{136}$, l'existence ${ }^{137}$, l'existence de Dieu ${ }^{138}$, l'experimentum ${ }^{139}$, la fin ${ }^{140}$, la foi ${ }^{141}$, la fonction judiciaire ${ }^{142}$, la génération ${ }^{143}$, la grammaire spéculative ${ }^{144}$, l'histoire ${ }^{145}$, l'image ${ }^{146}$, l'immanen-

S.J., Saint Thomas and the Lsomorphism of Human Knowledge and Its Proper Object, dans ScE, XIII, (1961), pp. 167-190; Anton C. PEGIS, Penitus Manet Ignotum, dans MSt, XXVII (1965), pp. 212-226; voir également note 159.

129. Voir plus bas, note 154 .

130. John N. DECK, St. Thomas Aquinas and the Language of Total Dependence, dans Dialogue, VI (1967), pp. 74-88.

131. SOEUR SAINTE-MARCELLE-D'AUVERGNE, De la matière du droit naturel, dans LTP, XXIII (1967), pp. 116-145; Philippe DELHAYE, Courte notice sur le droit naturel, dans SMR, V (1962), pp. 215-227.

132. Voir plus haut, note 122.

133. Voir plus bas, note 158 .

134. Benoît GARIÉPY, De l'utilité des erreurs, dans LTP, XIX (1963), pp. 179-205.

135. Andrew WEIGERT, S.J., A Cultural Aspect of Thomistic Etbics, dans RUO, 35 (1965), pp. $91^{*}-102 *$.

136. Louis LACHANCE, O.P., Note sur l'être, dans Etudes d'bistoire littéraire et doctrinale, 3e série (PIEM, XVII), Montréal-Paris, 1962, pp. 139-149; Etienne GILSON, Virtus essendi, dans MSt, XXVI (1964), pp. 1-11.

137. Voit ci-haut, note 116.

138. Harold J. JOHNSON, Why Five Ways? A Thesis and Some Alternatives, dans Arts libéraux... (voir plus haut note 52), pp. 1143-1154; voir encore ci-haut, note 101.

139. James S. STROMBERT, An Essay on Experimentum, dans LTP, XXIII (1967), pp. 76-115; XXIV (1968), pp. 99-138.

140. Guy DURAND, Les notions de fin intermédiaire et de fin secondaire dans la tradition thomiste, dans ScE, XXI (1969), pp. 371-402; David B. BURRELL, How God Achieves His Ends according to Saint Thomas Aquinas, dans $S C E$, XIV (1962), pp. 461-475; voir plus bas, note 170.

141. R. BELLEMARE, O.M.I., Credere. Note sur la définition thomiste, dans RUO, 30, I (1960), pp. $37^{*} .47^{*}$.

142. Voir plus bas, note 152 .

143. Oliva BLANCHETTE, The Order of Generation and Time in the Pbilo sopby of Saint Thomas Aquinas, dans LTP, XXII (1966), pp. 45-72.

144. Francis A. CUNNINGHAM, Speculative Grammar in St. Thomas Aquinas, dans LTP, XVII (1961), pp. 76-86.

145. Jean LANGLOIS, S.J., Premiers jalons d'une philosopbie thomiste de l'bistoire, dans ScE, XIV (1962), pp. 265-291; La catégorie moderne de l'bistoire et le thomisme, dans Dialogue, VII, 1 (1968), pp. 66-77.

146. Stanilas BRETON, Image, schème, concept, dans ScE, XV (1963), pp. $7-20$. 
$\mathrm{Ce}^{147}$, l'indéterminisme ${ }^{148}, \mathrm{l}$ ' $\left\langle\right.$ insight ${ }^{149}$, interprétations de textes particuliers ${ }^{150}$, la législation ${ }^{151}$, la loi naturelle ${ }^{152}$, les mathématiques ${ }^{153}$, la matière ${ }^{154}$, la métaphore ${ }^{155}$, le miracle ${ }^{156}$, la morale ${ }^{157}$, la musique ${ }^{158}$, la négation ${ }^{159}$, la névrose ${ }^{160}$, les ordres religieux ${ }^{161}$, la participation ${ }^{162}$, la pédagogie ${ }^{163}$, la philosophie chrétienne ${ }^{164}$, la philosophie de la nature ${ }^{165}$, la philo-

147. Germaine CROMP, Immanence et apprébension cbez saint Thomas, dans Dialogue, III (1964), pp. 235-247.

148. Voir plus bas, note 154 .

149. Albert R. JONSEN, «Insight», Thomism, and Contemporary Pbilosophy, dans RUO, 30, 2 (1960), pp. 102*-114*.

150. Vianney DÉCARIE, Les rédactions successives de la secunda via de «Contra Centiles》 I, 13, dans Dialogue, V (1966), pp. 205-216; Anton C. PEGIS, Saint Thomas and the Nicomachean Ethics: Some Reflections on «Summa Contra Gentiles》 III, 44 \& 5, dans MSt, XXV (1963), pp. 1-25; Robert J. MULVANEY, Political Wisdom. An Interpretation of Summa Theol. II-II, 50, dans MSt, XXXV (1973), pp. 294-305; T.M. ROBINSON, Averroes, Moerbeke, Aquinas, and a Crux in the De anima, dans MSt, XXXII (1970), pp. 340-344.

1.51. Voir plus bas, note 152 .

152. Joseph V. DOLAN, Natural Law and the Judicial Function, dans LTP. XVI (1960), pp. 94-141 ; Natural Law and Legislation, ibid., pp. 237-264.

153. Loui--Emile BLANCHET, La conception traditionnelle des matbématiques, dans LTP, XXIV (1968), pp. 161-171; voir encore plus bas, note 154.

154. Guy PICARD, Matière, contingence et indéterminisme chez saint Thomas, dans LTP, XXII (1966), pp. 197.233; FRÈRE AUGUSTIN-GABRIEL, Matière intelligible et matbématique, dans LTP, XVII (1961), pp. 173196 ; voir plus haut, note 116.

155. Ralph MINERNY, Metaphor and Anology, dans ScE, XVI (1964), pD. $273-289$.

156. Raymond LAFLAMME, Le miracle dans l'économie de la Parole, dans LTP, XX (1964), pp. 214-246.

157. Voir plus haut, note 125 .

158. Jasmin BOULAY, Le râle de la musique dans l'éducation, dans LTP. XVII (1961), po. 262-274.

159. Charles R. MACDONALD, The Role of Negation in Human Knowledge, dans $L T P$, XXI (1965), pp. 80-114.

160. Theresa M. CREM, A Thomistic Explanation of the Neurosis, dans LTP, XXIX (1968), pp. 294-300.

161. Caesarius NALPATHAMKALAM, Teacbing and Preaching Orders according to St. Thomas Aquinas, dans LTP, XXIII (1967), pp. 269-305.

162. Jean LANGLOIS, S.J., «Participation et causalité selon saint Thomas d'Aquin» par le P. Cornelio Fabro, dans ScE, XV (1963), pp. 277-290.

163. Voir plus bas, note 185 .

164. André NAUD, Le problème de la philosopbie chrétienne. Eléments d'une solution thomiste, dans SMR, III (1960), pp. 221-245; IV (1961). pp. 3-76.

165. Voir plus haut, note 118. 
sophie politique ${ }^{166}$, la piété filiale ${ }^{167}$, les premiers principes ${ }^{168}$, la prophétie ${ }^{169}$, la prudence ${ }^{170}$, la psychanalyse ${ }^{171}$, la recherche philosophique ${ }^{172}$, la sagesse ${ }^{173}$, le schème ${ }^{174}$, la science moyenne ne ${ }^{175}$, la sensation ${ }^{176}$, les sources patristiques ${ }^{177}$, la spiritualité ${ }^{178}$, la Somme de théologie ${ }^{179}$, le temps ${ }^{180}$, le 'tetragrammaton $^{181}$, la théologie ${ }^{182}$, l'universel ${ }^{183}$, la vérité ${ }^{184}$, la vertu ${ }^{185}$.

166. Léon CHARETTE, Pbilosopbie politique et métbode chez saint Thomas d'Aquin, dans RUO, 42, 1 (1972), pp. 83-96.

167. Joseph CHEN, Les doctrines chrétienne et confucéenne de ia piété filiale. dans LTP, XIX (1963), pp. 335-349.

168. Emmanuel TRÉPANIER, Sur deux premiers principes, dans LTP, XX (1964), pp. 132-141.

169. Jean RICHARD, Le processus psychologique de la révélation prophétique selon saint Thomas d'Aquin, dans LTP, XXIII (1967), pp. 42-75.

170. Paul MORISSET, S.J., Prudence et fin selon saint Thomas, dans SCE, XV (1963), pp. 73-98; 439-458; Bernard MORISSET, Le syllogisme prudentiel, dans LTP, XIX (1963), pp. 62-92.

171. Michael STOCK, Thomistic Thought and Contemporary Psychoanalysis, dans LTP, XIX (1963), pp. 350-360.

172. Voir plus haut, note 117.

173. Voir plus bas, note 182 .

174. Voir plus haut, note 146.

175. Louis RASOLO, S.J., Science moyenne et thomisme, dans ScE, XIX (1967), pp. 215-230.

176. Voir plus bas, note 178 .

177. Camille DOZOIS, Sources patristiques chez saint Thomas d'Aquin, dans RUO, 33, 1 (1963), pp. $28^{*}-48^{*} ; 33,2$ (1963), pp. $145^{*}-167^{*} ; 34.4$ (1964), pp. $231^{*} \cdot 241^{*} ; 35,2$ (1965), pp. 73*-90*.

178. Thomas FEELEY, "Spirituality》 and Sensation, dans LTP, XXI (1965), pp. 191-225.

179. Thomas-André AUDET, O.P., Approches bistoriques de la Summa theologiae, dans Etudes d'bistoire littéraire et doctrinale [3e série] (PIEM, XVIII), Montréal-Paris, 1962, pp. 7-29 ; André HAYEN, S.J., La structure de la somme théologique et Jésus, dans ScE, XII (1960), pp. 59-82.

180. Voir plus haut, note 143.

181. Armand MAURER, St. Thomas on the Sacred Name Tetragrammaton', dans MSt, XXXIV (1972), pp. 275-286.

182. Philip McSHANE, S.J., Theology and Wisdom, dans ScE, A1963Q, pp. 419-438; Anton C. PEGIS, St. Thomas and the Coherence of the Aristo. telian Theology, dans MSt, XXXV (1973), pp. 67-117.

183. Ronald MCARTHUR, Universal in praedicanda, Universal in causanda, dans LTP, XVIII (1962), pp. 59-95.

184. Michael J. LAPIERRE, S.J., Aquinas's Interpretation of Anselm's Definition of Truth, dans ScE, XVIII (1966), pp. 413-441; Armand MAURER, C.S.B., St. Thomas and Eternal Truth, dans MSt, XXXII (1970), pp. 91-107.

185. Jasmin BOULAY, Quelques notes à propos des vertus morales, I, dans $L T P$, XVI (1960), pp. 20-52; II, ibid., pp. 265-277; J. LEDUC, Implications 
Les travaux sur les maîtres dominicains postérieurs à saint Thomas ont porté sur des points de détail. L. Kennedy a tenté d'établir que Jean de Mechlin, et non Ulrich de Strasbourg, serait l'auteur du traité De bomine contenu dans la Summa de bono d'Ulrich ${ }^{186}$. B. Braswell croit qu'en plus de saint Thomas un second auteur, peut-être Boèce de Dacie, aurait utilisé la revision de la traduction des Seconds analytiques qui aurait très probablement été réalisée par Guillaume de Moerbeke ${ }^{187}$. E. B. Allen a étudié les positions de Hervaeus Natalis sur l'unicité de l'esse chez le Christ et l'infinité du pouvoir divin, positions qui confir-

théologiques de la vertu d'après saint Thomas d'Aquin, dans SMR, III, 2 (1960), pp. 201-219; IDEM, Implications pédagogiques de la vertu d'après saint Thomas d'Aquin, dans SMR, V (1962), pp. 229-245; VI (1963) , pp. 3-32. - Bien que l'histoire moderne et contemporaine du thomisme ne fasse pas partie de ce Bulletin, voici, pour ceux qu'intéresserait la question, quelques travaux aussi bien d'auteurs sympathiques à la survivance $\mathrm{du}$ thomisme que d'auteurs franchement partiaux et hostiles : Anton C. PEGIS, Thomism as a Pbilosophy (The McAuley Lectures, 1960), West Hartford, Conn.), 1961, pp. 15-30 ; Rodrigue NORMANDIN, O.M.I., Présence de saint Thomas d'Aquin, dans RUO, 30, 2 (1960), pp. 121-126; Mgr Régis JOLIVET, De l'actualité de saint Tbomas, dans ScE, XV (1963), pp. 325-349; Anton C. PEGIS, The Middle Ages and Philosopby. Some Reflections on the Ambivalence of Modern Scho. lasticism (The First James Roosevelt Bayley Lecture, Seton Hall University), Chicago, 1963, XVI-102 pp.; André DAGENAIS, Vingt-quatre défauts thomistes, Montréal, 1964, 206 pP.; Lucien LELIÈVRE, L'enseignement du tbomisme dans les collèges classiques, Montréal, 1965, 241 pp.; Etienne GILSON, On the Art of Misunderstanding Thomism (The McAuley Lectures, 1966), West Hartford (Conn.), 1966, pp. 33-44; Anton C. PEGIS, Catbolic Intellectualism at the Crossroad (The McAuley Lectures, 1966), West Hartford (Conn.), 1966, pp. 3-17; IDEM, Thomism 1966, ibid. 19-31; Jean RACETTE, Thomisme ou pluralisme? Réflexions sur l'enseignement de la pbilosophie. Préface de Jean LACROIX (Essais pour notre temps, 8), Bruges-Paris - Montréal, 1967, 129 pp.; Fernand VAN STEENBERGHEN, Le retour à saint Thomas a-t-il encore un sens aujourd'bui? (CAG 1967), Montréal-Paris, 1967, 61 pp.; Pierre THIBAULT, Savoir et pouvoir, philosophie thomiste et politique cléricale au XIXe siècle (Histoire et sociologie de la culture, 2), Québec, 1972, XXVIII-252 pp.

186. Leonard A. KENNEDY, C.S.B., The «De Homine 》 of Ulrich of Strasbourg, dans MSt, XXVII (1965), pp. 344-347.

187. Bruce BRASWELL, The Use of William of Moerbeke's Recension of the «Posterior Analytics》: a Second Instance, dans MSt, XXIV (1962), pp. 371-374. 
meraient que leur auteur fut un essentialiste plutôt qu'un existentialiste ${ }^{188}$.

Maitres séculiers. Peu de chercheurs canadiens ont consacré leurs efforts à des recherches sur les maitres séculiers des XIIIe-XIVe siècles. Il faut signaler le court aperçu de R. C. Dales sur les vues de Robert Grosseteste sur l'astrologie ${ }^{188}$, deux intéressantes études sur Dante, l'une de J. Pépin qui examine les notion et théorie de l'allégorie chez Dante, ses interprétations allégoriques de la culture païenne et de la Bible, enfin l'expression allégorique elle-même ${ }^{190}$, la seconde de $\mathrm{E}$. Gilson qui tente de faire de la lumière sur la nature de ces êtres que Dante à la suite de Virgile appelait des ombres ${ }^{191}$; une note dans laquelle A. Maurer et $W$. Dunphy montrent que les nouvelles oeuvres de Siger découvertes dans les ms Vienne, Bibliotbèque nationale lat. 2330 par A. Dondaine et L.-J. Bataillon sont de première importance pour le développement des études sigériennes ${ }^{192}$; une étude de A. C. Pegis de la question Utrum esse Deum possit fieri notum bomini alia via quam ex creaturis posée et discutée par Henri de Grand dans les a. 22, qu. 25, qu. 23 de sa Summa quaestionum ordinarium theologiae et dont la solution serait, dans l'idée de son auteur, la seule preuve adéquate conduisant à poser l'existence d'un rêve, unique dans son existence même ${ }^{193}$; un exposé par P.-E. Langevin de la position de Godefroid de Fontaines sur la

188. Elliott B. ALLEN, C.S.B., Hervaeus Natalis : an Early «Thomist» on the Notion of Being, dans MSt, XXII (1960), pp. 1-14.

189. Richard C. DALES, Robert Grosseteste's Vieus on Astrology, dans MSt, XXIX (1967), pp. 357-363.

190. Jean PÉPIN, Dante et la tradition de l'allégorie (CAG 1969), MontréalParis, 1970, 164 pp.

191. Étienne GILSON, Dante's Notion of a Shade: Purgatorio XXV, dans MSt, XXIX (1967), pp. 124-142.

192. William DUNPHY, and Armand MAURER, C.S.B., A Promising New Discovery for Sigerian Studies, dans MSt, XXIX (1967), pp. 364-369.

193. Anton C. PEGIS, Toward a New Way to God: Henry of Gbent, dans MSt, XXX (1968), pp. 226-247; XXXI (1969), pp. 93-116; Henry of Gbent and the New Way to God, III, dans MSt, XXXIII (1971), pp. 158-179. - Sur l'utilisation des théories de la connaissance d'Henri de Grand et de Duns Scot par Gianfrancesco Pico au XVIe siècle, voir Charles B. SCHMITT, Henry of Ghent, Duns Scotzes and Gianfrancesco Pico on Illtumination, dans MSt, XXV (1963), pp. 231-258. 
nature de la liberté humaine ${ }^{194}$; une synthèse, enfin, par J.A. Weisheipl de la vie et des oeuvres des Maîtres de Merton College Walter Burley, Thomas Bradwardine, William Heytesbury, John Dumbleton, Richard Swineshead, ainsi que de celles de Guillaume d'Ockham sans l'apport duquel les contributions à la logique et à la physique des premiers ne peuvent être appréciées avec justesse et exactitude ${ }^{19}$.

Institut d'études médiévales

Université de Montréal

194. Paul-Émile LANGEVIN, S.J., Nécessité ou liberté chez Godefroid da Fnntaines, dans ScE, XII (1960), pp. 175-203.

195. James A. WEISHEIPL, Ockbam and Some Mertonians, dans MSt, XXX (1968), pp. 163-213. 